\title{
Gambling in Contests modelled with diffusions
}

\author{
Han Feng • David Hobson
}

the date of receipt and acceptance should be inserted later

\begin{abstract}
In the Seel-Strack contest (Seel and Strack, 2013), $n$ agents each privately observe an independent copy of a drifting Brownian motion which starts above zero, and is absorbed at zero. Each agent chooses when to stop the process she observes, and the winner of the contest is the agent who stops her Brownian motion at the highest value. The objective of each agent is to maximise her probability of winning the contest. Seel \& Strack derived a Nash equilibrium under a joint feasibility condition on the drift and the number of players.

We consider a generalisation of the Seel-Strack contest in which the observed processes are independent copies of some time-homogeneous diffusion. This naturally leads us to consider the problem in cases where the analogue of the feasibility condition is violated. We solve the problem via a change of scale and a Lagrangian method. Unlike in the Seel-Strack problem it turns out that the optimal strategy may involve a target distribution which has an atom, and the rule used for breaking ties becomes important.
\end{abstract}

Keywords Seel-Strack contest; Nash equilibrium; Randomized strategies; Lagrangian method; Diffusions; Skorokhod embedding problem

\section{Introduction}

Motivated by casino tournaments, Seel and Strack (2013) introduced a model of a gambling contest between agents in which the aim of each agent is to maximise the probability that her return is highest amongst the set of all

Han Feng

Department of Statistics, University of Warwick, Coventry, CV4 7AL, UK. E-mail: H.Feng@warwick.ac.uk

David Hobson

Department of Statistics, University of Warwick, Coventry, CV4 7AL, UK. Tel.: +44 (0)24 7615 0396, Fax: +44 (0)24 7652 4532. E-mail: D.Hobson@warwick.ac.uk 
agents. This problem provides a stylised model for a competition between fund managers, the most successful of whom will be given funds to invest over the next period or will get a bonus. The problem described in Seel and Strack (2013) is simple to state but has very rich and subtle solutions.

In the Seel \& Strack paper each agent privately observes an independent copy of a process $Y=\left(Y_{t}\right)_{t>0}$, where $Y$ is a drifting Brownian motion absorbed at zero with initial value $y_{0}>0$, constant drift $\mu$ and constant diffusion coefficient $\sigma>0$. The agent chooses a stopping time $\tau$, and the stopped value $Y_{\tau}$ forms her entry into the contest. The agent who stops at the highest value wins the contest, and the objective of each agent is to maximise the probability of winning. In a contest between $n$ agents, Seel \& Strack imposed a feasibility condition

$$
\mu<\log \left(1+\frac{1}{n-1}\right) \frac{\sigma^{2}}{2 y_{0}} .
$$

With this condition in force, they showed that there exists a Nash equilibrium within the class of bounded stopping rules. A feature of the Nash equilibrium is that it involves a randomised strategy, and the aim of each agent is to choose a stopping time $\tau$ such that the final value of the stopped process $Y_{\tau}$ has distribution $F$. The Nash equilibrium is unique in the sense that the target distribution $F$ is unique, but in general there are many stopping times $\tau$ such that $Y_{\tau}$ has law $F$. (One of the insights of Seel and Strack (2013) is that the problem can be reduced to a search over distributions, and then that optimal stopping times can be identified as solutions of the Skorokhod embedding problem (Skorokhod, 1965).) It turns out that $F$ is the distribution function of a continuous random variable (in particular it is atom-free) and has a density which is strictly positive on a bounded interval, and is zero elsewhere.

Seel and Strack (2013) solved the problem by giving a unique candidate value function for the problem, and then proving this candidate is a martingale under an optimal stopping rule for each agent. We adopt a different method of proof based on a Lagrangian sufficiency theorem. Moreover, our first step is to transform the problem into natural scale. This allows us to consider more general models for the observed process, beyond drifting Brownian motion, such that $Y$ is a time-homogeneous diffusion. The change of scale method explains the origin of the condition (1) and motivates us to study the problem in which (1) fails.

The effect of using the scale function is to transform the original contest into a simpler contest in which the observed processes are martingales. To illustrate this procedure, consider the model used in Seel and Strack (2013). Let $Y$ be a drifting Brownian motion with non-zero drift $\mu$ and diffusion coefficient $\sigma$, started at $y_{0}>0$ and such that if $Y$ hits zero then it is absorbed there. Then the scale function is $s(y)=\frac{\sigma^{2}}{2 \mu}-\frac{\sigma^{2}}{2 \mu} e^{-2 \mu y / \sigma^{2}}$ (we have chosen a normalisation such that $s(0)=0)$ and $X=s(Y)$ is a martingale diffusion with starting value $x_{0}=s\left(y_{0}\right)$ which solves $d X_{t}=\sigma\left(1-\frac{2 \mu X_{t}}{\sigma^{2}}\right) d W_{t}$, at least until it first hits zero, which is an absorbing point. Observe that the state space of $X$ is $[0, s(\infty))$. 
Then it is easy to check that the condition (1) imposed by Seel \& Strack is equivalent to $s(\infty)>n x_{0}$. We are interested in the cases where $s(\infty)<n x_{0}$, which are not covered in Seel and Strack (2013).

We introduce the mathematical model of this contest in Section 2. We start with a contest in which the observed process is any non-negative timehomogeneous diffusion. Then we explain how this contest is equivalent to a contest in which the observed process is a diffusion in natural scale. In Section 3, we derive the Nash equilibrium using a technique based on the Lagrangian sufficiency theorem. We will see that the choice of method used to break ties matters. We solve the problem for two canonical ways of breaking ties. Finally, in Section 4, we discuss two examples of $Y$ and give explicit expressions for the Nash equilibrium and an optimal stopping rule in each case.

Our results show that the strategy which the agent should use in a Nash equilibrium is determined by both the mechanism for the breaking of ties and the value of the upper bound of the state space of the diffusion in natural scale. Moreover, there exist multiple Nash equilibria if the way to break the ties has been improperly chosen. There are close links between the problem and an all-pay auction with a bid cap (Che and Gale (1998)).

Acknowledgement: The authors would like to thank an anonymous referee, in particular for his advice on relating this work to extant economic literature.

\section{The model}

In the contest, there are $n$ participants with labels $i \in I=\{1,2, \ldots, n\}$. Agent $i$ privately observes an independent copy $Y^{i}$ of a non-negative timehomogeneous diffusion process $Y=\left(Y_{t}\right)_{t>0}$ with constant initial value $Y_{0}=y_{0}$. Assume the state space of $Y$ is an interval $J$ with endpoints $\left\{0, r \in\left(y_{0}, \infty\right]\right\}$.

If $Y$ can reach an endpoint in finite time then we assume that the endpoint is absorbing. Further, to exclude degeneracies, we assume that $\lim _{t \uparrow \infty} Y_{t}$ exists, almost surely (and then $\left.\lim _{t \uparrow \infty} Y_{t} \in\{0, r\}\right)$ and that $\mathbb{P}\left(\lim _{t \uparrow \infty} Y_{t}=0\right)>0$. (We return to this point in Remark 1 below.) Examples include Brownian motion with drift, absorbed at zero, and exponential Brownian motion, provided that the parameters are such that the process does not diverge to infinity, see Example 2.

Let $\mathcal{F}_{t}^{Y^{i}}=\sigma\left(\left\{Y_{s}^{i}: s<t\right\}\right)$ and set $\mathbb{F}^{Y^{i}}=\left(\mathcal{F}_{t}^{Y^{i}}\right)_{t \geq 0}$. The space of strategies for agent $i$ is the space of $\mathbb{F}^{Y^{i}}$-stopping times $\tau^{i}$. Without loss of generality we restrict attention to $\tau^{i} \leq H_{0}^{i}=\inf \left\{t \geq 0: Y_{t}^{i}=0\right\}$. Note that $\tau^{i}$ is not necessarily assumed to be finite, that is, agent $i$ may choose to never stop the process $Y^{i}$, in which case her entry is taken to be $\lim _{t \uparrow \infty} Y_{t}$. Note also that agent $i$ observes her own process $Y^{i}$, but not $Y^{j}$ for $j \neq i$; nor does she observe the stopping times chosen by the other agents.

The winner of the contest is the one who stops at the highest value and she wins unit reward, that is, $\forall i \in I$, agent $i$ wins 1 if she stops at a time $\tau^{i}$ such that $Y_{\tau^{i}}^{i}>Y_{\tau^{j}}^{j} \forall j \neq i$. If there are $k$ agents who stop at the equal highest value then these agents each win $\theta(k)$, where $\theta(\cdot):\{1,2, \ldots, n\} \mapsto[0,1]$ 
is some decreasing (deterministic) function with $\theta(1)=1$. Therefore agent $i$ with stopping value $Y_{\tau^{i}}^{i}$ receives payoff

$$
\theta(k) \cdot \mathbf{1}_{\left\{Y_{\tau^{i}}^{i}=\max _{j \in I} Y_{\tau j}^{j}\right\}},
$$

where $k=\left|\left\{i \in I: Y_{\tau^{i}}^{i}=\max _{j \in I} Y_{\tau^{j}}^{j}\right\}\right|$.

In general, there are two canonical choices of the ways to break the ties. One choice is to divide the prize evenly, that is, to set $\theta(k)=1 / k$. This is equivalent to randomly breaking ties. Another choice is to reward only outright wins, so that no one wins if there is more than one player who stops at the highest value and $\theta(k)=\mathbf{1}_{\{k=1\}}$. (There is a third, less natural choice which is to set $\theta(k)=1$, or equivalently to reward joint winners with the full prize. In this case the problem is degenerate, and a Nash equilibrium is obtained by all agents stopping immediately, $\tau^{i}=0$.) We will give explicit solutions for the first two cases in Section 3.

Suppose that $Y$ is a solution of the stochastic differential equation (SDE) $d Y_{t}=a\left(Y_{t}\right) d W_{t}+b\left(Y_{t}\right) d t$ where $b$ is continuous and $a$ is continuous and positive on the interior of $J$. Let $s=s(y)$ be the scale function of $Y$. Then $s$ is a strictly increasing solution of $a(y)^{2} s^{\prime \prime}(y)+2 b(y) s^{\prime}(y)=0$. In general, $s(J)$ is an interval with endpoints $\{L, U\}$ with $-\infty \leq L<U \leq \infty$ and there are four sub-cases depending on whether either $L$ or $U$ is finite or not. In fact, by the Rogozin trichotomy, our assumption that $\lim Y_{t}$ exists rules out the case that $s(J)=\mathbb{R}$, and the assumption that $\mathbb{P}\left(\lim _{t \uparrow \infty} Y_{t}=0\right)>0$ rules out the case that $L=-\infty$. Since $s$ is only determined up to affine transformation we may set $s(0)=0$, and then $s(J)$ is an interval with endpoints $\{0, U\}$ where $U$ may be finite or infinite. We could also insist that $s\left(y_{0}\right)=1$ but we do not choose to do so.

Let $X=s(Y)$. Then $X$ is a diffusion in natural scale on $s(J)$ with starting value $x_{0}=s\left(y_{0}\right)$, see Rogers and Williams (2000, Chapter V.7) or Karatzas and Shreve (1991, Section 5.5). Set $\mathbb{F}^{X}$ to be the natural filtration of $X$ (and by extension $\mathbb{F}^{X^{i}}$ to be the natural filtration of $\left.X^{i}=s\left(Y^{i}\right)\right)$. Clearly $\tau$ is a $\mathbb{F}^{Y}$-stopping time if and only if $\tau$ is an $\mathbb{F}^{X}$ stopping time.

Since $s(\cdot)$ is a continuous, strictly increasing function, the payoff of agent $i$ with stopping value $Y_{\tau^{i}}^{i}$ can be rewritten as $\theta(k) \cdot \mathbf{1}_{\left\{X_{\tau^{i}}^{i}=\max _{j \in I} X_{\tau^{j}}^{j}\right\}}$, where $X_{\tau^{i}}^{i}=s\left(Y_{\tau^{i}}^{i}\right)$ and $k=\left|\left\{i \in I: X_{\tau^{i}}^{i}=\max _{j \in I} X_{\tau^{j}}^{j}\right\}\right|$. This implies the equivalence between the two contests in which players privately observe $Y^{i}$ and $X^{i}$ respectively, and the optimal stopping rule $\tau^{i}$ is the same for both contests. In particular, if we have a Nash equilibrium for which $\tau^{i}$ is optimal for the process $X^{i}$, then we also have a Nash equilibrium for the processes $Y^{i}$. Hence, without loss of generality we may reduce the problem to the case in which the observed process is a copy of a local martingale diffusion.

One of the insights of Seel and Strack (2013) is that since the payoffs to the agents are determined by the distribution of $X_{\tau^{i}}^{i}$ rather than the stopping time $\tau^{i}$ itself, the problem of choosing the optimal stopping time $\tau^{i}$ can be reduced to a problem of finding the optimal distribution $F_{X}^{i} \equiv F^{i}$ of $X_{\tau^{i}}^{i}$. 
Then, once we have found the optimal target distribution $F^{i}$, the remaining work is to verify that there exists $\tau^{i}$ such that $X_{\tau^{i}}^{i}$ has law $F_{X}^{i}$. It follows that $Y_{\tau^{i}}^{i}$ has law $F_{Y}^{i}=F_{X}^{i} \circ s$.

The problem of finding $\tau^{i}$ such that $X_{\tau^{i}}^{i}$ has distribution $F_{X}^{i}$ is related to the classical Skorokhod embedding problem (Skorokhod, 1965).

Since $X$ is a diffusion in natural scale, by the Dambis-Dubins-Schwarz Theorem (e.g. Rogers and Williams (2000), Theorem 34.1, p. 64) $X$ can be expressed as a time change of Brownian motion. Then $X_{t}=B_{\Gamma_{t}}$ for some Brownian motion $B$ with $B_{0}=x_{0}$ and an increasing functional $\Gamma_{t}=[X]_{t}$. Then, if $F$ is the distribution function of any random variable on $[0, U]$ with mean $x_{0}$, there exists a $\mathbb{F}^{B}$-stopping time $\rho$ such that $\rho \leq \inf \left\{v: B_{v}=\right.$ 0 or $\left.B_{v}=U\right\}$ and $B_{\rho}$ has distribution $F$. Such a $\rho$ is known as a solution of the Skorokhod embedding problem. In general there are many such solutions. Then if we take $\tau=\Gamma^{-1} \circ \rho$ we find $X_{\tau}=B_{\rho} \sim F$ and $\tau$ is also an embedding of $F_{Y}=F_{X} \circ s$ in $Y$. Moreover $\tau$ is a $\mathbb{F}^{Y}$-stopping time. If $\rho<\inf \{v$ : $B_{v}=0$ or $\left.B_{v}=U\right\}$ then it follows that $\tau$ is finite, and more generally $\tau \leq \inf \left\{v: X_{v}=0\right.$ or $\left.X_{v}=U\right\}=\inf \left\{v: Y_{v}=0\right.$ or $\left.Y_{v}=r\right\} \leq \infty$.

If $U<\infty$ then $\left(X_{t \wedge \inf \left\{v: X_{v}=0 \text { or } X_{v}=U\right\}}\right)_{t \geq 0}$ is a martingale and every candidate target distribution $F^{X}$ for $X$ must have mean $x_{0}$. However, $\left(X_{t \wedge \inf \left\{v: X_{v}=0 \text { or } X_{v}=U\right\}}\right)_{t \geq 0}$ is a priori only a local martingale if $U=\infty$. Nonetheless, it is a supermartingale, and hence cannot explode to $U=\infty$, and $\lim _{t} X_{t}=0=\lim _{t} Y_{t}$ almost surely. In this case, for any distribution $\tilde{F}$ with mean strictly less than $x_{0}$ with associated embedding $\tilde{\tau}$, there exists $(F, \tau)$ such that $F$ has mean $x_{0}$, $F \leq \tilde{F}$ and $X_{\tau} \sim F$. Clearly $\tau$ dominates $\tilde{\tau}$ as a strategy, which means such $(\tilde{F}, \tilde{\tau})$ must not be optimal.

Remark 1 For the duration of this remark relax the assumptions that $\lim _{t \uparrow \infty} Y_{t}$ exists and that $\mathbb{P}\left(\lim _{t \uparrow \infty} Y_{t}=0\right)>0$. If $\lim _{t \uparrow \infty} Y_{t}$ does not exist then $s(J)=\mathbb{R}$. If $\lim _{t \uparrow \infty} Y_{t}$ exists and is equal to zero almost surely, then $s(0)=-\infty$. In either case lim $\sup _{t \uparrow \infty} Y_{t}=r$ with probability 1 , and then any stopping rule which involves stopping at $Y_{t}=\hat{y}$ for $\hat{y}<r$ can be improved upon by waiting until $Y$ hits $(\hat{y}+r) / 2$. Hence, either the optimal strategy is to wait until $Y$ hits $r$ (either in finite time, or in the limit) or there is no optimal strategy.

In terms of the process in natural scale $\lim \sup _{t \uparrow \infty} Y_{t}=r$ is equivalent to $\lim \sup _{t \uparrow \infty} X_{t}=U$ which is the case if and only if $L=-\infty$. This is why we have excluded the case.

Example 1 (Drifting Brownian motion.) Let $\tilde{Y}$ be a drifting Brownian motion so that $\tilde{Y}_{t}=y_{0}+\mu t+\sigma W_{t}$, where $y_{0}>0, \mu \neq 0$ and $\sigma>0$ are all constants. Let $\tilde{H}_{0}=\inf \left\{u: \tilde{Y}_{u}=0\right\}$ and let $Y_{t}=\tilde{Y}_{t \wedge \tilde{H}_{0}}$. Then $Y=\left(Y_{t}\right)_{t \geq 0}$ is a drifting Brownian motion absorbed at zero, and has state space $J=[0, \infty)$. The scale function of both $\tilde{Y}$ and $Y$ is $s(y)=\frac{\sigma^{2}}{2 \mu}-\frac{\sigma^{2}}{2 \mu} e^{-2 \mu y / \sigma^{2}}$. Since $s(0)=0$ and $s(\infty) \leq \infty, \lim _{t \uparrow \infty} Y_{t}$ exists and $\mathbb{P}\left(\lim _{t \uparrow \infty} Y_{t}=0\right)>0$. In particular, if $\mu>0$ then $s(\infty)=\frac{\sigma^{2}}{2 \mu}<\infty$ and $\mathbb{P}\left(\lim _{t \uparrow \infty} Y_{t}=0\right)=1-s\left(y_{0}\right) / s(\infty)=e^{-2 \mu y_{0} / \sigma^{2}}$, whereas if $\mu<0$ then $s(\infty)=\infty$ and thus $\mathbb{P}\left(\lim _{t \uparrow \infty} Y_{t}=0\right)=1$. 
Drifting Brownian motion absorbed at zero is the process considered in the original Seel and Strack paper, although they do not map it into natural scale.

Example 2 (Exponential Brownian motion.) Now suppose $Y$ is an exponential Brownian motion, so that $Y$ solves $d Y_{t}=\mu Y_{t} d t+\sigma Y_{t} d W_{t}$, subject to $Y_{0}=$ $y_{0}>0$, where $y_{0}, \mu$ and $\sigma$ are all constants. $Y$ has state space $(0, \infty)$ and scale function $s(y)=y^{\kappa} / \kappa$ for $\kappa \neq 0$ and $s(y)=\ln y$ for $\kappa=0$, where $\kappa=1-2 \mu / \sigma^{2}$. We assume $\kappa>0$ to ensure that $s(0)$ is finite so that $\lim _{t \uparrow \infty} Y_{t}$ exists and $\mathbb{P}\left(\lim _{t \uparrow \infty} Y_{t}=0\right)>0$. If $\kappa \leq 0$ then $\mathbb{P}\left(\limsup _{t \uparrow \infty} Y_{t}=\infty\right)=1$ and this example is degenerate.

The remainder of the paper is devoted to finding a Nash equilibrium for the problem in the sense of a family of the optimal target distributions $\left(F^{i}\right)_{i \in I}$ of $\left(X_{\tau^{i}}^{i}\right)_{i \in I}$. The family $\left(F^{i}\right)_{i \in I}$ is a Nash equilibrium, if for each $i \in I$, given the other agents choose to stop at $\tau^{j}$ such that $X_{\tau^{j}}^{j} \sim F^{j}$, the optimal stopping rule of agent $i$ is to choose any $\tau^{i}$ such that $X_{\tau^{i}}^{i} \sim F^{i}$. We will say a Nash equilibrium is symmetric if $F^{i}$ does not depend on $i$, and we will say that a Nash equilibrium has no atoms in $[0, U)$ if each $F^{i}$ has no atoms in $[0, U)$.

Given that the contest is symmetric in the sense that each agent observes a martingale process started from the same level $x_{0}$, it seems natural to search for Nash equilibria which are symmetric. Then, a simple argument over rearranging mass show that provided $\theta(k)<1$ for some $k \geq 2$ it is never optimal for $k$ agents to put mass at a same point $x \in(0, U)$ - any of them could benefit by modifying the target distribution to put a proportion $N /(N+1)$ of this mass at $\left(x+N^{-2}\right)$ and a proportion $1 /(N+1)$ at $\left(x-N^{-1}\right)$ (where $N$ is a sufficiently large number) — and thus it is possible to deduce that any optimal solution puts no mass at any point which belongs to $(0, U)$. Notice that this argument does not apply to the case where the mass point is $U$. In fact, in Section 3 we will see that in some cases it is indeed optimal to put mass at the upper bound $U$.

Remark 2 The fact that the Nash equilibrium has no atom at zero relies on the fact that the situation is symmetric. If the observed processes have different starting points, then the Nash equilibrium may have masses at zero for some agents. In that case, for a Nash equilibrium, at least one agent must put no mass at zero. We will only consider the symmetric case.

Remark 3 When $U \geq n x_{0}$ (for example when $U=\infty$ ) the solution to our problem can be identified with the solution provided in Seel and Strack (2013). In this case there exists a unique symmetric, atom-free Nash equilibrium. Moreover, Seel \& Strack proved that in this case any Nash equilibrium has the property that it is symmetric and atom-free. Thus the Nash equilibrium that has been found is the unique Nash equilibrium for our problem.

The novel part of our solution, beyond the fact that we consider general diffusion processes, is that in the case $U<n x_{0}$ we identify a Nash equilibrium. This equilibrium may depend on the method used to break ties, but provided this method has been chosen sensibly then there is a symmetric Nash equilibrium, which may involve an atom at $U$. By analogy with the case studied 
in Seel and Strack we expect this to be the unique Nash equilibrium, but our focus is on proving that such equilibria exist.

\section{Equilibrium distribution}

From Section 2, we know that the original contest can be reduced to a new contest in which each agent $i$ privately observes an independent copy $X^{i}$ of a continuous martingale diffusion process $X=\left(X_{t}\right)_{t \geq 0}$ where $X_{0}=x_{0}>0$ is a constant. The process $X$ is defined on $[0, U]$, where $x_{0}<U \leq \infty$. Agent $i$ who stops at $X_{\tau^{i}}^{i}$ receives payoff

$$
\theta(k) \cdot \mathbf{1}_{\left\{X_{\tau^{i}}^{i}=\max _{j \in I} X_{\tau^{j}}^{j}\right\}},
$$

where $k=\left|\left\{i \in I: X_{\tau^{i}}^{i}=\max _{j \in I} X_{\tau^{j}}^{j}\right\}\right|$.

In this section we explicitly discuss the two canonical choices of $\theta(\cdot), \theta(k)=$ $1 / k$ and $\theta(k)=\mathbf{1}_{\{k=1\}}$ (and briefly, $\theta(k)=1$ in Remark 9 ). These correspond to the cases where ties are broken randomly, and only outright wins earn the prize respectively (and thirdly the case where all joint winners are rewarded with the full prize). We give the candidate Nash equilibrium solution and then verify the candidate Nash equilibrium using the Lagrangian sufficiency theorem. We will see that the two different choices of $\theta(\cdot)$ give us different results.

Theorem 1 1) Suppose $U \geq n x_{0}$, and recall $\theta(k) \leq 1$ for all $k$. Then there exists a symmetric Nash equilibrium for the problem that is atom-free and $X_{\tau^{i}}^{i}$ has law $F(x)$, where for $x \geq 0$

$$
F(x)=\min \left\{\sqrt[n-1]{\frac{x}{n x_{0}}}, 1\right\} .
$$

2) Suppose $x_{0}<U<n x_{0}$.

i) If $\theta(k)=\mathbf{1}_{\{k=1\}}$ then there exists a symmetric Nash equilibrium for the problem that has no atoms in $[0, U)$ but an atom at $U$ of size $\frac{n x_{0}-U}{(n-1) U}$, and $X_{\tau^{i}}^{i}$ has law $F$ such that for $0 \leq x<U$

$$
F(x)=\frac{n\left(U-x_{0}\right)}{(n-1) U} \sqrt[n-1]{\frac{x}{U}} .
$$

ii) If $\theta(k)=1 / k$ then there exists a symmetric Nash equilibrium for the problem that has no atoms in $[0, U)$ but an atom at $U$ of size $p=1-\phi$, where $\phi \in\left(0, \sqrt[n-1]{U /\left(n x_{0}\right)}\right)$ solves $\Phi(\varphi)=0$. Here $\Phi(\varphi)=x_{0}\left(1-\varphi^{n}\right)-U(1-\varphi)$.

Further, $X_{\tau^{i}}^{i}$ has law $F$ such that for $0 \leq x<U$

$$
F(x)=\min \left\{\sqrt[n-1]{\frac{x}{n x_{0}}}, \phi\right\} .
$$


Proof Let $\mathcal{A}$ be the set of pairs $(F, p)$ where $F:[0, U) \mapsto[0, \infty)$ is a nondecreasing function null at 0 , and $p \in \mathbb{R}^{+}$. An element of $\mathcal{A}$ is identified with a measure $\nu$ on $[0, U]$ such that $F(x)=\nu([0, x])$ and $p=\nu(\{U\})$. Let $\mathcal{A}\left(1, x_{0}\right)$ be the subset of $\mathcal{A}$ identified with probability measures with mean $x_{0}$. Then $\mathcal{A}\left(1, x_{0}\right)$ is given by

$$
\mathcal{A}\left(1, x_{0}\right)=\left\{(F, p) \in \mathcal{A}: \lim _{x \uparrow U} F(x)+p=1 \text { and } \int_{[0, U)} x F(d x)+U p=x_{0} .\right\}
$$

We seek a symmetric Nash equilibrium that has no atoms in $[0, U)$. Since there are no atoms in $[0, U)$, a Nash equilibrium is identified with a pair $\left(G^{*}, q^{*}\right) \in \mathcal{A}\left(1, x_{0}\right)$ such that $\forall(G, q) \in \mathcal{A}\left(1, x_{0}\right)$

$$
\begin{aligned}
\int_{[0, U)} G^{*}(x)^{n-1} G^{*}(d x)+\left[\sum_{k=1}^{n} \theta(k) C_{n-1}^{k-1}\left(q^{*}\right)^{k-1}\left(1-q^{*}\right)^{n-k}\right] q^{*} \\
\geq \int_{[0, U)} G^{*}(x)^{n-1} G(d x)+\left[\sum_{k=1}^{n} \theta(k) C_{n-1}^{k-1}\left(q^{*}\right)^{k-1}\left(1-q^{*}\right)^{n-k}\right] q .
\end{aligned}
$$

Fix agent $i$. Suppose that the other players all choose $(F, p)$ as their target measure. Then agent $i$ aims to choose a feasible law of $X_{\tau^{i}}^{i}$, which corresponds to a pair $(G, q) \in \mathcal{A}\left(1, x_{0}\right)$, to solve

$$
\max _{(G, q) \in \mathcal{A}} \int_{[0, U)} F(x)^{n-1} G(d x)+\left[\sum_{k=1}^{n} \theta(k) C_{n-1}^{k-1} p^{k-1}(1-p)^{n-k}\right] q
$$

subject to $\int_{[0, U)} x G(d x)+U q=x_{0}$ and $\int_{[0, U)} G(d x)+q=1$. Introducing multipliers $\lambda$ and $\gamma$ for the two constraints, the Lagrangian for the optimisation problem (2) is then

$$
\begin{aligned}
\mathcal{L}_{F, p}(G, q ; \lambda, \gamma)= & \int_{[0, U)}\left[F(x)^{n-1}-\lambda x-\gamma\right] G(d x) \\
& +\left[\sum_{k=1}^{n} \theta(k) C_{n-1}^{k-1} p^{k-1}(1-p)^{n-k}-\lambda U-\gamma\right] q+\lambda x_{0}+\gamma .
\end{aligned}
$$

Now we state a variant of the Lagrangian sufficiency theorem for our problem.

Proposition 1 If there exist $G^{*}, q^{*}, \lambda^{*}$ and $\gamma^{*}$ such that $\left(G^{*}, q^{*}\right) \in \mathcal{A}\left(1, x_{0}\right)$, $G^{*}$ is continuous on $[0, U)$ and

$$
\mathcal{L}_{G^{*}, q^{*}}\left(G^{*}, q^{*} ; \lambda^{*}, \gamma^{*}\right) \geq \mathcal{L}_{G^{*}, q^{*}}\left(G, q ; \lambda^{*}, \gamma^{*}\right)
$$

for all $(G, q) \in \mathcal{A}$, then $\left(G^{*}, q^{*}\right)$ is a symmetric Nash equilibrium that has no atoms in $[0, U)$. 
Proof If $(G, q) \in \mathcal{A}\left(1, x_{0}\right)$ then using the definition of the Lagrangian,

$\int_{[0, U)} G^{*}(x)^{n-1} G(d x)+\left[\sum_{k=1}^{n} \theta(k) C_{n-1}^{k-1}\left(q^{*}\right)^{k-1}\left(1-q^{*}\right)^{n-k}\right] q=\mathcal{L}_{G^{*}, q^{*}}\left(G, q ; \lambda^{*}, \gamma^{*}\right)$.

Then, under the hypotheses of the proposition,

$$
\begin{aligned}
\int_{[0, U)} G^{*}(x)^{n-1} G^{*}(d x)+ & {\left[\sum_{k=1}^{n} \theta(k) C_{n-1}^{k-1}\left(q^{*}\right)^{k-1}\left(1-q^{*}\right)^{n-k}\right] q^{*}=\mathcal{L}_{G^{*}, q^{*}}\left(G^{*}, q^{*} ; \lambda^{*}, \gamma^{*}\right) } \\
\geq \mathcal{L}_{G^{*}, q^{*}}\left(G, q ; \lambda^{*}, \gamma^{*}\right) & =\int_{[0, U)} G^{*}(x)^{n-1} G(d x)+\left[\sum_{k=1}^{n} \theta(k) C_{n-1}^{k-1}\left(q^{*}\right)^{k-1}\left(1-q^{*}\right)^{n-k}\right] q .
\end{aligned}
$$

Return to the proof of Theorem 1.

1) Suppose $U \geq n x_{0}$. On $[0, \infty)$ let $G^{*}(x)=\min \left\{\sqrt[n-1]{x /\left(n x_{0}\right)}, 1\right\}, q^{*}=$ $0, \lambda^{*}=1 /\left(n x_{0}\right)$ and $\gamma^{*}=0$. It is immediate that $\left(G^{*}, q^{*}\right)$ correspond to a distribution with mean $x_{0}$, and so it remains to verify (4) for the given multipliers.

Since $\theta(k) \leq 1$ for all $k$ we have that

$$
\sum_{k=1}^{n} \theta(k) C_{n-1}^{k-1}\left(q^{*}\right)^{k-1}\left(1-q^{*}\right)^{n-k} \leq \sum_{k=1}^{n} C_{n-1}^{k-1}\left(q^{*}\right)^{k-1}\left(1-q^{*}\right)^{n-k}=(q+(1-q))^{n}=1
$$

and then

$$
\begin{gathered}
\mathcal{L}_{G^{*}, q^{*}}\left(G, q ; \lambda^{*}, \gamma^{*}\right) \leq \int_{[0, U)}\left[G^{*}(x)^{n-1}-\lambda^{*} x-\gamma^{*}\right] G(d x)+\left(1-\lambda^{*} U-\gamma^{*}\right) q+\lambda^{*} x_{0}+\gamma^{*} \\
=\int_{\left(n x_{0}, U\right)}\left(1-\frac{x}{n x_{0}}\right) G(d x)+\left(1-\frac{U}{n x_{0}}\right) q+\frac{1}{n} \leq \frac{1}{n}=\mathcal{L}_{G^{*}, q^{*}}\left(G^{*}, q^{*} ; \lambda^{*}, \gamma^{*}\right) .
\end{gathered}
$$

Thus there exists a symmetric, atom-free Nash equilibrium.

2) Suppose $x_{0}<U<n x_{0}$.

i) Set $\theta(k)=\mathbf{1}_{\{k=1\}}$. On $[0, \infty)$ let $G^{*}(x)=\left[\frac{n\left(U-x_{0}\right)}{(n-1) U} \sqrt[n-1]{\frac{x}{U}}\right] \cdot \mathbf{1}_{\{x<U\}}+$ $\mathbf{1}_{\{x \geq U\}}, q^{*}=\frac{n x_{0}-U}{(n-1) U}, \lambda^{*}=\left[\frac{n\left(U-x_{0}\right)}{(n-1) U}\right]^{n-1} U^{-1}$ and $\gamma^{*}=0$. Now we verify that for these multipliers (4) holds and that $\left(G^{*}, q^{*}\right) \in \mathcal{A}\left(1, x_{0}\right)$. The latter property follows from the explicit form of $G^{*}$ and $q^{*}$. For the former, since $\theta(k)=\mathbf{1}_{\{k=1\}}$,

$$
\begin{aligned}
\mathcal{L}_{G^{*}, q^{*}} & \left(G, q ; \lambda^{*}, \gamma^{*}\right) \\
& =\int_{[0, U)}\left[G^{*}(x)^{n-1}-\lambda^{*} x-\gamma^{*}\right] G(d x)+\left[\left(1-q^{*}\right)^{n-1}-\lambda^{*} U-\gamma^{*}\right] q+\lambda^{*} x_{0}+\gamma^{*} \\
& =\left[\frac{n\left(U-x_{0}\right)}{(n-1) U}\right]^{n-1} \frac{x_{0}}{U}=\mathcal{L}_{G^{*}, q^{*}}\left(G^{*}, q^{*} ; \lambda^{*}, \gamma^{*}\right) .
\end{aligned}
$$

Thus there exists a symmetric Nash equilibrium that has no atoms in $[0, U)$. 
ii) Set $\theta(k)=1 / k$. On $[0, \infty)$ let $G^{*}(x)=\min \left\{\sqrt[n-1]{x /\left(n x_{0}\right)}, \phi\right\} \cdot \mathbf{1}_{\{x<U\}}+$ $\mathbf{1}_{\{x>U\}}, q^{*}=1-\phi, \lambda^{*}=1 /\left(n x_{0}\right)$ and $\gamma^{*}=0$, where $\phi \in(0,1)$ solves $\Phi(\varphi)=0$ and $\Phi(\varphi)=x_{0} \varphi^{n}-U \varphi+U-x_{0}$. Because $\Phi(0)=U-x_{0}>0$, $\Phi(1)=0, \Phi^{\prime}(0)=-U<0, \Phi^{\prime}(1)=n x_{0}-U>0$ and $\Phi$ is convex on $(0, \infty)$, there exists a unique solution to $\Phi(\varphi)=0$ such that $\varphi \in(0,1)$. Moreover, since $\Phi(\phi)=x_{0} \phi^{n}-U \phi+U-x_{0}=0$ and $\phi \in(0,1), \frac{U}{n x_{0}}=\frac{1-\phi^{n}}{n(1-\phi)}=$ $\frac{1}{n}\left(1+\phi+\phi^{2}+\cdots+\phi^{n-1}\right)>\phi^{n-1}$ and thus $\phi<\sqrt[n-1]{U /\left(n x_{0}\right)}$.

Now we verify that for these multipliers (4) holds and that $\left(G^{*}, q^{*}\right) \in$ $\mathcal{A}\left(1, x_{0}\right)$. Again, the latter fact follows from the explicit form of $G^{*}$ and $q^{*}$. Then, since $q^{*} \neq 0$ and $\theta(k)=1 / k$, and since $C_{n-1}^{k-1} / k=C_{n}^{k} / n$,

$$
\begin{aligned}
\sum_{k=1}^{n} \theta(k) C_{n-1}^{k-1}\left(q^{*}\right)^{k-1}\left(1-q^{*}\right)^{n-k} & =\frac{1}{n q^{*}} \sum_{k=1}^{n} C_{n}^{k}\left(q^{*}\right)^{k}\left(1-q^{*}\right)^{n-k}=\frac{1}{n q^{*}}\left[1-\left(1-q^{*}\right)^{n}\right] \\
& =\frac{1}{n(1-\phi)}\left(1-\phi^{n}\right)=\frac{U}{n x_{0}}
\end{aligned}
$$

and we have

$$
\begin{aligned}
\mathcal{L}_{G^{*}, q^{*}}\left(G, q ; \lambda^{*}, \gamma^{*}\right) \\
\quad=\int_{[0, U)}\left[G^{*}(x)^{n-1}-\lambda^{*} x-\gamma^{*}\right] G(d x)+\left[\frac{U}{n x_{0}}-\lambda^{*} U-\gamma^{*}\right] q+\lambda^{*} x_{0}+\gamma^{*} \\
\quad=\int_{\left(n x_{0} \phi^{n-1}, U\right)}\left(\phi^{n-1}-\frac{x}{n x_{0}}\right) G(d x)+\frac{1}{n} \leq \frac{1}{n}=\mathcal{L}_{G^{*}, q^{*}}\left(G^{*}, q^{*} ; \lambda^{*}, \gamma^{*}\right) .
\end{aligned}
$$

So that (4) holds. Thus there exists a symmetric Nash equilibrium that has no atoms in $[0, U)$.

Remark 4 In the case where $U$ cannot be reached in finite time, if the optimal target law places mass on $U$ then this corresponds to the optimal stopping rule $\tau=\infty$ for that part of the sample space where $X_{\tau}=U$.

Remark 5 There are strong parallels between our model and an all-pay auction in which the stopped value of the process corresponds to the auction bid, so that the choice over distributions for the stopped value of the process corresponds to the choice over distributions for the bid size. The upper bound on the state space of the stochastic process in natural scale corresponds to the bid cap.

It follows that the same Lagrangian methods can be applied to the all-pay auction. The cost from making a bid then enters directly into the objective function and the agent chooses any probability distribution on $\mathbb{R}^{+}$. In contrast, in the context of gambling in contests there is no cost associated with the bid in the objective function, but the constraint that the target probability distribution has mean $x_{0}$ introduces an extra term into the Lagrangian.

More precisely, consider a symmetric all-pay auction with a cap $m$ on bids, where $0<m \leq \infty$. We assume that there are $n$ bidders in the auction, and 
all bidders have the same valuation $v$ of the prize. The Lagrangian for this problem is

$$
\begin{aligned}
\mathcal{L}_{F, p}^{\mathrm{apa}}(G, q ; \gamma)= & \int_{[0, m)}\left[v F(x)^{n-1}-x-\gamma\right] G(d x) \\
& +\left[v \sum_{k=1}^{n} \theta(k) C_{n-1}^{k-1} p^{k-1}(1-p)^{n-k}-m-\gamma\right] q+\gamma .
\end{aligned}
$$

Comparing with (3) we see that modulo a factor of $v$ representing the size of the winnings and a relabelling of parameters, the main difference is that in (5) the multiplier $\lambda$ on the bid level is set to $1 / v$.

In the case where $m \geq v$ or the case where $m \in(v / n, v)$ and $\theta(k)=1 / k$, the equilibrium distribution in this all-pay auction is exactly the same as the equilibrium distribution in our model with $m=U$ and $v=n x_{0}$. In the case where $m \in(0, v)$ and $\theta(k)=\mathbf{1}_{\{k=1\}}$, these two equilibrium distributions are also the same with $m=U$ and $v=U\left[\frac{(n-1) U}{n\left(U-x_{0}\right)}\right]^{n-1}$.

Remark 6 Expanding on the previous remark, for the most standard tie-breaking rule, i.e. $\theta(k)=1 / k$, Theorem 1 shows that if $x_{0}<U<n x_{0}$, then there is a "hole" in the support of the equilibrium distribution. Specifically, in equilibrium, players stop with positive probability on $\left[0, n x_{0} \phi^{n-1}\right]$, players stop with zero probability on $\left(n x_{0} \phi^{n-1}, U\right)$, and players stop at $U$ with probability $1-\phi$. Similar equilibrium distributions with holes have been found in all-pay auctions with bid caps, e.g., Che and Gale (1998), Dechenaux et al. (2006) or Szech (2011) and also in wars of attrition, e.g., Hendricks et al. (1988) and Damiano et al. (2012).

Remark 7 If $x_{0}<U<n x_{0}, \theta(k)=\mathbf{1}_{\{k=1\}}$ (so that only outright wins are rewarded) and all other agents follow strategies which yield the optimal target distribution stated in Case 2i) of Theorem 1, then whatever stopping rule agent $i$ chooses her expected payoff is equal to $\frac{x_{0}}{U}\left[\frac{n\left(U-x_{0}\right)}{(n-1) U}\right]^{n-1}$. In the other two cases, Cases 1) and 2ii) of the theorem, if other agents use the Nash equilibrium strategy, then the agent achieves the same expected payoff as the optimal strategy, provided she puts no mass in $\left(n x_{0}, U\right]$ or $\left(n x_{0} \phi^{n-1}, U\right)$ respectively.

Remark 8 The choice of the tie-breaking rule is crucial in determining the Nash equilibrium, at least in cases where the upper bound is sufficiently small. This phenomena is also a feature of some variants of the all-pay auction in which optimal bid distributuions include an atom, see for example Che and Gale (1998), Dechenaux et al. (2006), Cohen and Sela (2007), and Szech (2011).

Szech (2011) studies a two-player all-pay auction in which each player is restricted to choose her bid from the interval $[0, m]$. She introduces an asymmetry whereby it is assumed that if both bidders submit the same bid, bidder 1 wins with probability $\alpha \in[0,1]$, otherwise bidder 2 wins. In this auction, 
Szech shows that the Nash equilibrium depends on the choice of tie-braking rule via $(\alpha, m)$, and takes one of three distinct forms.

Remark 9 If the way of breaking ties has not been chosen appropriately, then there might exist multiple Nash equilibria. (In the context of all-pay auctions, Cohen and Sela (2007) show that there may be multiple symmetric equilibria even in the standard case $\theta(k)=1 / k$, but there the phenomena arises from the discreteness of the set of possible bids.)

Take $\theta(k) \equiv 1$ (in which tied winners all win the full prize) as an example in the context of this paper. It is clear that the stopping rule such that every agent stops immediately is a symmetric Nash equilibrium and that the associated target distribution consists of unit mass at $x_{0} \in[0, U)$. Moreover, there exists a symmetric Nash equilibrium that has no atoms in $[0, U)$. This can be proved similarly to the proof of Theorem 1 . In fact, if $U \geq n x_{0}$ then there exists a symmetric Nash equilibrium for the problem that has no atoms and $X_{\tau^{i}}^{i}$ has law $F(x)$, where $F(x)=\min \left\{\sqrt[n-1]{x /\left(n x_{0}\right)}, 1\right\}$ for $x \geq 0$; if $U<n x_{0}$ then there exists a symmetric Nash equilibrium for the problem that has no atoms in $[0, U)$ but an atom at $U$ of size $(1-\hat{\phi})$, and $X_{\tau^{i}}^{i}$ has law $F$ such that $F(x)=\min \left\{\sqrt[n-1]{x /\left(n x_{0}\right)}, \hat{\phi}\right\}$ for $0 \leq x<U$. Here $\hat{\phi} \in(0,1)$ solves $\hat{\Phi}(\varphi)=0$, where $\hat{\Phi}(\varphi)=U \varphi^{n}-n U \varphi+n\left(U-x_{0}\right)$.

\section{Examples}

In this section, we give explicit expressions for the optimal target distribution and associated stopping time. The optimal stopping time is based on the Azéma-Yor solution of the Skorokhod embedding problem (Azéma and Yor (1979)). Note that any other solution for the Skorokhod embedding problem (see Hobson (2011); Obłój (2004) for a survey) can also be used to construct an optimal strategy, but the Azéma-Yor solution is both relatively simple and quite concrete.

\subsection{Drifting Brownian motion}

Suppose the diffusion process $Y$ is a drifting Brownian motion absorbed at zero and solves $d Y_{t}=\mu d t+\sigma d W_{t}$ where $Y_{0}=y_{0}>0, \mu \neq 0$ and $\sigma>0$ are all constants. Set $\gamma=2 \mu / \sigma^{2} \neq 0$. The scale function of $Y$ is $s(y)=\frac{1}{\gamma}-\frac{1}{\gamma} e^{-\gamma y}$. Let $X=s(Y)$; then $X$ is a diffusion in natural scale on $[0, U)$ with starting value $x_{0}=s\left(y_{0}\right)$, and $U=s(\infty)$. If $\mu>0$ then $U=s(\infty)=1 / \gamma$, else if $\mu<0$ then $U=s(\infty)=\infty$.

Seel and Strack (2013) discussed the case where $U>n x_{0}$, which is equivalent to the condition (1) i.e. $\gamma<\frac{1}{y_{0}} \log \frac{n}{n-1}$. Here we discuss the general case. 
1) Suppose $U \geq n x_{0}$, that is, suppose $\gamma \leq \frac{1}{y_{0}} \log \frac{n}{n-1}$. Recall that $\theta(k) \leq 1$ for all $k$, then by Case 1) of Theorem 1, the optimal distribution of $X_{\tau}$ is $F(x)=\min \left\{\sqrt[n-1]{x /\left(n x_{0}\right)}, 1\right\}$ for $x \geq 0$. Define

$$
\psi(x)=\frac{1}{1-F(x)} \int_{[x, \infty)} y F(d y)
$$

for $x \leq \inf \{x: F(x)=1\}$ and $\psi(x)=x$ otherwise. Then $\psi$ is the barycentre function. Thus $\psi(x)=x$ for $x \geq n x_{0}$, and for $0 \leq x<n x_{0}$,

$$
\psi(x)=x_{0} \frac{\left(1-\left(\frac{x}{n x_{0}}\right)^{n /(n-1)}\right)}{\left(1-\left(\frac{x}{n x_{0}}\right)^{1 /(n-1)}\right)} .
$$

The Azéma-Yor embedding of $F$ in Brownian motion started at $x_{0}=(1-$ $\left.e^{-\gamma y_{0}}\right) / \gamma$ is $\tau=\inf \left\{t \geq 0: \psi\left(X_{t}\right) \leq \bar{X}_{t}\right\}$, where $\bar{X}_{t}=\sup _{s<t} X_{s}$.

We want to re-interpret this solution in terms of the drifting Brownian motion $Y$. Set $F_{Y}=F \circ s$ so that $F_{Y}(y)=0$ for $y \leq 0$,

$$
F_{Y}(y)=\left(\frac{\left(1-e^{-\gamma y}\right)}{n\left(1-e^{-\gamma y_{0}}\right)}\right)^{1 /(n-1)} \quad \text { for } y \in\left(0,-\frac{1}{\gamma} \log \left(1-n\left(1-e^{-\gamma y_{0}}\right)\right)\right)
$$

and $F_{Y}(y)=1$ for $y \geq-\frac{1}{\gamma} \log \left(1-n\left(1-e^{-\gamma y_{0}}\right)\right)$.

Then, since $s$ is increasing, $\tau$ can be rewritten as $\tau=\inf \left\{t \geq 0: \Psi\left(Y_{t}\right) \leq \bar{Y}_{t}\right\}$ where $\Psi(y):=s^{-1}(\psi(s(y)))$ is given by

$$
\Psi(y)=-\frac{1}{\gamma} \log \left(1-\left(1-e^{-\gamma y_{0}}\right) \frac{\left\{1-\left(\frac{\left(1-e^{-\gamma y}\right)}{n\left(1-e^{-\gamma y_{0}}\right)}\right)^{n /(n-1)}\right\}}{\left\{1-\left(\frac{\left(1-e^{-\gamma y}\right)}{n\left(1-e^{-\gamma y_{0}}\right)}\right)^{1 /(n-1)}\right\}}\right)
$$

if $0 \leq y<-\frac{1}{\gamma} \log \left(1-n\left(1-e^{-\gamma y_{0}}\right)\right)$ and $\Psi(y)=y$ otherwise.

2) Suppose $x_{0}<U<n x_{0}$, that is, suppose $\gamma>\frac{1}{y_{0}} \log \frac{n}{n-1}>0$. Assume agents receive no reward if they are a tied winner so that $\theta(k)=\mathbf{1}_{\{k=1\}}$. By Case 2i) of Theorem 1, the optimal distribution of $X_{\tau}$ has law $F$ such that $F(x)=\frac{n\left(U-x_{0}\right)}{(n-1) U} \sqrt[n-1]{\frac{x}{U}}$ for $0 \leq x<U$ and $F(x)=1$ for $x \geq U$. Then by the definition of the barycentre function $\psi$ in $(6), \psi(x)=x$ for $x>U$ and

$$
\psi(x)=\frac{x_{0}(n-1)-x\left(1-\frac{x_{0}}{U}\right) \sqrt[n-1]{\frac{x}{U}}}{n-1-n\left(1-\frac{x_{0}}{U}\right) \sqrt[n-1]{\frac{x}{U}}} \text { for } x \in[0, U] .
$$

Recalling that $U=1 / \gamma$ and substituting $s(y)=\frac{1}{\gamma}\left(1-e^{-\gamma y}\right), s^{-1}(x)=$ $-\frac{1}{\gamma} \log (1-\gamma x)$ and (10) into the expressions $F_{Y}=F \circ s$ and $\Psi(y)=s^{-1}(\psi(s(y)))$ 
yields

$$
\begin{aligned}
F_{Y}(y) & =\frac{n}{n-1} e^{-\gamma y_{0}}\left(1-e^{-\gamma y}\right)^{1 /(n-1)} \\
\Psi(y) & =y_{0}-\frac{1}{\gamma} \log \left(\frac{n-1-\left(n-1+e^{-\gamma y}\right) \sqrt[n-1]{1-e^{-\gamma y}}}{n-1-n e^{-\gamma y_{0}} \sqrt[n-1]{1-e^{-\gamma y}}}\right),
\end{aligned}
$$

for $0 \leq y<\infty$. In particular, if $n=2$ then

$$
\Psi(y)=y+\frac{1}{\gamma} \log \left(e^{\gamma\left(y_{0}+y\right)}-2 e^{\gamma y}+2\right) .
$$

Note that $\gamma y_{0}>\log 2$ by hypothesis, so that the term inside the logarithm is positive.

Then $\tau=\inf \left\{t \geq 0: \Psi\left(Y_{t}\right) \leq \bar{Y}_{t}\right\}$ is the Azéma-Yor optimal stopping rule for the original contest. We have that $\lim _{y \uparrow \infty} F_{Y}(y)<1$, so there is a non-zero probability that $\tau=\infty$ and that the agent achieves an infinite entry into the contest.

3) Again suppose $\gamma>\frac{1}{y_{0}} \log \frac{n}{n-1}$ but assume $\theta(k)=1 / k$. The optimal distribution $F$ of $X_{\tau}$ is given by Case 2ii) of Theorem 1 as $F(x)=$ $\min \left\{\sqrt[n-1]{\frac{x}{n x_{0}}}, \phi\right\}$ for $0 \leq x<U$ and $F(x)=1$ for $x \geq U$, where $U=1 / \gamma$ and $\phi \in\left(0, \sqrt[n-1]{\frac{U}{n x_{0}}}\right)$ solves $\Phi(\varphi)=0$ with $\Phi(\varphi)=x_{0}\left(1-\varphi^{n}\right)-U(1-\varphi)$. Then, by the definition (6) of the barycentre function, for $0 \leq x \leq n x_{0} \phi^{n-1}$, $\psi(x)$ is given by (7), for $n x_{0} \phi^{n-1}<x \leq U$ we have $\psi(x)=U$, and for $x>U$, $\psi(x)=x$

Let $F_{Y}=F \circ s$. Then for $y>0, F_{Y}(y)=\min \left\{\phi,\left[\frac{1-e^{-\gamma y}}{n\left(1-e^{-\gamma y_{0}}\right)}\right]^{1 /(n-1)}\right\}$.

Let $\Psi(y)=s^{-1}(\psi(s(y)))$. Then $\Psi(y)$ is given by (9) if $y \in\left[0, s^{-1}\left(n x_{0} \phi^{n-1}\right)\right]$ with $s^{-1}\left(n x_{0} \phi^{n-1}\right)=-\frac{1}{\gamma} \log \left(1-n \phi^{n-1}\left(1-e^{-\gamma y_{0}}\right)\right)$ and $\Psi(y)=\infty$ otherwise. The Azéma-Yor optimal stopping rule for the original contest is $\tau=$ $\inf \left\{t \geq 0: \Psi\left(Y_{t}\right) \leq \bar{Y}_{t}\right\}$. Again there is a non-zero probability that $\tau=\infty$ and that the agent achieves an infinite entry into the contest.

\subsection{Exponential Brownian motion}

The above methods extend easily to any non-negative time-homogenenous diffusion with state space an interval with endpoints $\{0, r \in(0, \infty]\}$ provided the scale function $s$ satisfies $L=s(0)>-\infty$. Then we can normalise $s$ so that $s(0)=0$. Depending on the value of $U=s(r)$ we are in one of the cases of Theorem 1. In each case, for the diffusion in natural scale the optimal target law is given as in the theorem, and by the formula (6) for the barycentre $\psi$ we can construct an optimal stopping rule. The barycentre and the stopping time are exactly as in Section 4.1. Finally, it remains to interpret these stopping times as stopping times for the original process, and only at this stage do the calculations look different to the drifting Brownian motion case. 
As a further example, now suppose agents privately observe independent copies of an exponential Brownian motion $Y$. Suppose $Y$ is a solution of $d Y_{t}=$ $\mu Y_{t} d t+\sigma Y_{t} d W_{t}$, where $Y_{0}=y_{0}>0$, and $y_{0}, \mu$ and $\sigma$ are all constants. In light of the discussion in Example 2, we assume $\mu \in\left(-\infty, \sigma^{2} / 2\right)$. The scale function of $Y$ is $s(y)=y^{\kappa} / \kappa$, where $\kappa=1-2 \mu / \sigma^{2}$ and $\kappa>0$. Let $X=s(Y)$; then $X$ is a diffusion in natural scale on $(0, \infty)$ with starting value $x_{0}=s\left(y_{0}\right)$.

In this case $U=s(\infty)=\infty$ and trivially $U \geq n x_{0}$. Provided that $\theta(k) \leq 1$ for all $k$, then by Case 1) of Theorem 1, the optimal distribution of $X_{\tau}$ is $F(x)=\min \left\{\sqrt[n-1]{x /\left(n x_{0}\right)}, 1\right\}$ for $x \geq 0$ and the barycentre function $\psi$ is the same as shown in (7). The the optimal law of $Y$ is $F_{Y}=F \circ s$ where for $y \geq 0$

$$
F_{Y}(y)=\min \left\{1,\left(\frac{y}{y_{0}}\right)^{\kappa /(n-1)} n^{-1 /(n-1)}\right\}
$$

Using the Azéma-Yor embedding one solution is $\tau=\inf \left\{t \geq 0: \Psi\left(Y_{t}\right) \leq \bar{Y}_{t}\right\}$, where

$$
\Psi(y)=y_{0}\left(1-\left(\frac{y^{\kappa}}{n y_{0}^{\kappa}}\right)^{n /(n-1)}\right)^{1 / \kappa}\left(1-\left(\frac{y^{\kappa}}{n y_{0}^{\kappa}}\right)^{1 /(n-1)}\right)^{-1 / \kappa}
$$

if $0 \leq y<y_{0} n^{1 / \kappa}$ and $\Psi(y)=y$ otherwise. Note that $\lim _{y \uparrow y_{0} n^{1 / \kappa}} \Psi(y)=y_{0} n^{1 / \kappa}$ and hence $\tau \leq \inf \left\{u: Y_{u}=y_{0} n^{1 / \kappa}\right\}$.

\section{A Derivation of the equilibrium distribution}

This section is devoted to the derivation of the optimal multipliers, and the candidate Nash equilibrium distributions given in Theorem 1.

Recall the definition of the Lagrangian $\mathcal{L}_{F, p}(G, q ; \lambda, \gamma)$ for the optimisation problem (2). Let $l(p)=\sum_{k=1}^{n} \theta(k) C_{n-1}^{k-1} p^{k-1}(1-p)^{n-k}-\lambda U-\gamma$ and $L_{F}(x)=F(x)^{n-1}-\lambda x-\gamma$, then

$$
\mathcal{L}_{F, p}(G, q ; \lambda, \gamma)=\int_{[0, U)} L_{F}(x) G(d x)+l(p) q+\lambda x_{0}+\gamma .
$$

In order to have a finite optimal solution we require $L_{F}(x) \leq 0$ on $[0, U)$ and $l(p) \leq 0$. Let $\mathcal{D}_{F}$ be the set of $(\lambda, \gamma)$ such that $\mathcal{L}_{F, p}(\cdot, \cdot ; \lambda, \gamma)$ has a finite maximum. Then $\mathcal{D}_{F}$ is defined by

$$
\mathcal{D}_{F}=\left\{(\lambda, \gamma): L_{F}(x) \leq 0 \forall x \in[0, U) \text { and } l(p) \leq 0\right\} .
$$

In order to reach the maximum value, we require $G(d x)=0$ when $L_{F}(x)<0$ and $q=0$ if $l(p)<0$. This means that for $(\lambda, \gamma) \in \mathcal{D}_{F}$ the maximum of $\mathcal{L}_{F, p}(\cdot, \cdot ; \lambda, \gamma)$ occurs at $\left(G^{*}, q^{*}\right)$ such that $G^{*}(d x)=0$ when $L_{F}(x)<0$ and $q^{*}=0$ when $l(p)<0$. Conversely we expect that $G^{*}(d x)>0$ when $L_{F}(x)=0$ and $q^{*}>0$ when $l(p)=0$. If the Nash equilibrium is symmetric then we must have $G^{*}(x)=F(x)$ and $q^{*}=p$, which means $L_{G^{*}}(x)=0$ at least when $G^{*}(d x)>0$ and $l\left(q^{*}\right)=0$ when $q^{*}>0$.

Observe that $0 \leq F^{n-1}(0) \leq \gamma$ so that if $(\lambda, \gamma) \in \mathcal{D}_{F}$ then $\gamma$ is non-negative. Because $L_{G^{*}}(x)=G^{*}(x)^{n-1}-\lambda x-\gamma$, we have $G^{*}(x)=\sqrt[n-1]{\lambda x+\gamma}$ at least when $G^{*}(d x)>0$. Since $G^{*}$ is non-decreasing and not constant we must have $\lambda>0$. Set $a=\inf \left\{x: G^{*}(x)>0\right\}$ and $b=\sup \left\{x: G^{*}(x)<\left(1-q^{*}\right)\right\}$. Since we are searching for $G^{*}(x)$ which has no atom on $[0, U)$, we must have $G^{*}(x)=\sqrt[n-1]{\lambda x+\gamma}$ on the whole of the interval $[a, b)$. 
Since $G^{*}$ has no atom on $[0, U), G^{*}(a)=0$ and hence $\lambda a+\gamma=0$, and by the nonnegativity of $a$ and $\gamma$ and the positivity of $\lambda$ it follows that $\gamma=0=a$. Thus $G^{*}(x)=\sqrt[n-1]{\lambda x}$ on $[0, b)$ for some $\lambda>0$ and $b \leq U$ which we must find. Further, we must find $q^{*} \in[0,1)$ that solves $l\left(q^{*}\right)=0$ when $q^{*} \neq 0$.

For a feasible solution, $G^{*}$ and $q^{*}$ should satisfy $\int_{[0, U)} x G^{*}(d x)+U q^{*}=x_{0}$ and $\int_{[0, U)} G^{*}(d x)+q^{*}=1$. Thus to get $G^{*}$ and $q^{*}$ we should solve the following system of equations

$$
\left\{\begin{array}{l}
x_{0}=\int_{[0, b)} x d(\sqrt[n-1]{\lambda x})+U q=b \sqrt[n-1]{\lambda b}-\frac{n-1}{n \lambda}(\lambda b)^{\frac{n}{n-1}}+U q=\frac{b}{n} \sqrt[n-1]{\lambda b}+U q, \\
1=\int_{[0, b)} d(\sqrt[n-1]{\lambda x})+q=\sqrt[n-1]{\lambda b}+q, \\
l(q)=\sum_{k=1}^{n} \theta(k) C_{n-1}^{k-1} q^{k-1}(1-q)^{n-k}-\lambda U=0, \text { if } q \neq 0 .
\end{array}\right.
$$

If $q=0$ then from (11) we obtain $b=n x_{0}$ and $\lambda=1 /\left(n x_{0}\right)$. Thus $q=0$ is a feasible solution if $b=n x_{0} \leq U$ and is not a feasible solution otherwise. Next we search for non-zero $q$ that is feasible.

i) Set $\theta(k)=\mathbf{1}_{\{k=1\}}$. Then the third equation in (11) can be reduced to $(1-q)^{n-1}-\lambda U=$ 0 if $q \neq 0$. Thus for $q \neq 0$, (11) can be reduced to

$\lambda=\frac{(1-q)^{n-1}}{U} ; b=\frac{(1-q)^{n-1}}{\lambda} ; x_{0}=\frac{(1-q)^{n}}{n \lambda}+U q=\frac{U(1-q)}{n}+U q=\frac{U+(n-1) U q}{n}$.

This gives us the optimal $q^{*}=\frac{n x_{0}-U}{(n-1) U}$ and then $\lambda^{*}=\left[\frac{n\left(U-x_{0}\right)}{(n-1) U}\right]^{n-1} U^{-1}$ and $b^{*}=U$. This gives us the $G^{*}$ given in the statement of the theorem.

ii) Set $\theta(k)=1 / k$. Observe that we have shown $\sum_{k=1}^{n} \frac{1}{k} C_{n-1}^{k-1} q^{k-1}(1-q)^{n-k}=$ $\frac{1}{n q}\left[1-(1-q)^{n}\right]$ if $q \neq 0$ in the proof of Theorem 1. For $q \neq 0$, (11) can be reduced

$$
\begin{aligned}
& \lambda=\frac{1-(1-q)^{n}}{n U q} ; \quad b=\frac{(1-q)^{n-1}}{\lambda} \\
& x_{0}=\frac{(1-q)^{n}}{n \lambda}+U q=\frac{U q(1-q)^{n}}{1-(1-q)^{n}}+U q=\frac{U q}{1-(1-q)^{n}} .
\end{aligned}
$$

Let $\phi=1-q$ then (13) can be rewritten as $\Phi(\phi)=0$, where $\Phi(\varphi)=x_{0} \varphi^{n}-U \varphi+U-x_{0}$.

Since $\Phi$ is convex on $(0,1)$, we can find that there exists an solution $\phi$ to $\Phi(\varphi)=0$ such that $\phi \in(0,1)$ if and only if $U<n x_{0}$. Moreover, such solution is unique. Denote $\phi^{*}$ by this solution if it exists.

Therefore, if $U<n x_{0}$ then $q^{*}=1-\phi^{*}$ and then $\lambda^{*}=1 /\left(n x_{0}\right)$ and $b^{*}=n x_{0}\left(1-q^{*}\right)^{n-1}$ using (12)

\section{References}

Azéma, J., Yor, M., 1979. Une solution simple au problème de Skorokhod, in: Seminaire de Probabilites XIII, Springer, pp. 90-115.

Che, Y.-K., Gale, I., 1998. Caps on political lobbying. American Economic Review, 88(3):643-651.

Cohen, C., Sela, A., 2007. Contests with ties. The B.E: Journal of Theoretical Economics, $7(1): 1-16$.

Dechenaux, E., Kovenock, D., Lugovskyy, V, 2006. Caps on bidding in all-pay auctions: Comments on the experiments of A. Rapoport and W. Amaldoss. Journal of Economic Behavior and Organization, 61(2):276-283.

Damiano, E., Hao, L., Suen, W., 2012. Optimal deadlines for agreements. Theoretical Economics, 7(2):357-393.

Hendricks, K., Weiss, A., Wilson, C., 1988. The war of attrition in continuous time with complete information. International Economic Review, 29(4):663-680. 
Hobson, D.G., 2011. The Skorokhod embedding problem and model independent bounds for option prices, in: Paris-Princeton Lecture Notes on Mathematical Finance 2010, Springer, pp. 267-318.

Karatzas, I., Shreve, S.E., 1991. Brownian Motion and Stochastic Calculus, 2nd ed. Graduate Texts in Mathematics, Springer.

Obłój, J., 2004. The Skorokhod embedding problem and its offspring. Probab. Surveys 1:321392.

Rogers, L.C.G., Williams, D., 2000. Diffusions, Markov Processes and Martingales: Volume 2, Itô Calculus. Cambridge Mathematical Library, Cambridge University Press.

Skorokhod, A.V., 1965. Studies in the theory of random processes. Addison-Wesley, Reading, Mass.

Szech, N., 2011. Tie-breaks and bid-caps in all-pay auctions. Mineo.

Seel, C., Strack, P., 2013. Gambling in Contests. Journal of Economic Theory, 148(5):20332048. 